\title{
Implementación de un método numérico para la solución de una ecuación del tráfico
}

\author{
E. J. López-Sánchez \\ Facultad de Filosofía y Letras, Universidad Nacional Autónoma de México, \\ Circuito Interior. Ciudad Universitaria, s/n. 04510. Ciudad de México, México. \\ e-mail:lsej@unam.mx \\ P. E. Olivera \\ Seminario de Estudios Urbanos Críticos, Facultad de Filosofía y Letras, Universidad Nacional Autónoma de México, \\ Circuito Interior. Ciudad Universitaria, s/n. 04510. Ciudad de México, México. \\ e-mail: oliverap@unam.mx
}

Received 11 February2020; accepted 7 April 2020

\begin{abstract}
Los estudiantes de licenciatura y posgrado en ciencias tienen conocimientos muy heterogéneos de herramientas computacionales para resolver problemas matemáticos. Generalmente, los profesores desconocen de todos los programas existentes, así que deben mediar entre las herramientas que ellos manejen y las que dominan los alumnos, para que el curso avance sin dificultad y sin detenerse a enseñar un solo tipo de instrumento. Se aborda esta problemática utilizando la siguiente metodología. Se explica el algoritmo general que resuelve el problema, y se ejecuta en Scilab, un lenguaje de programación fácil de aprender, de libre distribución y mediante el cual se grafican los resultados directamente. Se implementa en el modelo de tráfico vehicular de Greenberg. Se utilizan datos de variables de tráfico medidos en una vialidad de la Ciudad de México como condiciones iniciales y de frontera para el modelo.
\end{abstract}

Descriptores: Tráfico vehicular; modelo Greenberg modificado; diferencias finitas; Gauss-Seidel.

Undergraduate and graduate science students have very heterogeneous knowledge of computational tools to solve mathematical problems. Teachers usually ignore how to handle all existing programs, so they must mediate between the tools they know and those that students dominate so that the course progresses without difficulty and without stopping to teach a single type of instrument. This problem is addressed using the following methodology. The general algorithm that solves the problem is explained, it is implemented on Scilab, a programming language that is easy to learn, freely distributed, and in which the results are plotted directly. It is implemented in the Greenberg vehicle traffic model. Data measured in a Mexico City street of the vehicular traffic variables, namely, speed, vehicular density and vehicular flow are used as initial and boundary conditions for the model.

Keywords: Vehicular traffic; modified Greenberg model; finite differences; Gauss-Seidel.

PACS: 47.40.-x; 45.05.+x; 45.70.Vn

\section{Introducción}

Actualmente, las computadoras ayudan a resolver problemas matemáticos aplicados en todas las áreas del conocimiento que involucran sistemas de ecuaciones con una gran cantidad de incógnitas. Desafortunadamente, los planes de estudio de licenciaturas en ciencias como Física, Química, Biología, Ciencias de la Tierra y algunas Ingenierías (salvo licenciaturas como Ciencias de la computación, Informática o Ingenierías en Computación) contemplan muy pocas asignaturas de computación, pues priorizan el abordaje de los problemas utilizando una herramienta matemática en varios semestres en cursos como Matemáticas avanzadas de la Física, Métodos Matemáticos, Ecuaciones Diferenciales Parciales, Funciones Especiales, entre otras.

Al examinar los planes de estudio de las licenciaturas en Física de algunas de las universidades más importantes de México, se encuentra que en promedio se consideran solo dos materias, con carácter de obligatorias, concernientes a computación o métodos numéricos en el transcurso de ocho a diez semestres de duración de la carrera. Ciertamente, existen más de dos asignaturas de este tipo en la curricula de las carreras
DOI: https://doi.org/10.31349/RevMexFisE.17.165

mencionadas, pero son optativas y la mayoría de los alumnos prefiere aquellas relacionadas con los temas en los que desean especializarse. Algunos de estos planes de estudio se pueden consultar en [1-9].

Esta escasez de materias obligatorias de métodos numéricos provoca que los grupos de estudiantes sean totalmente heterogéneos en cuanto a nociones de las herramientas computacionales; desde los alumnos que únicamente saben usar hojas de cálculo, hasta los que tienen conocimientos avanzados de programación en lenguajes de alto nivel. El profesor que imparta alguna cátedra de computación o física computacional debe mediar al momento de dar su clase. No puede darle preferencia a quienes solo saben usar las hojas de cálculo, porque aquellos que saben programar perderían el interés en la clase, pero tampoco debe priorizar a los que dominan la programación computacional, porque los otros desertarían, ya que generalmente los cursos de física computacional y métodos numéricos a nivel superior no se planearon para enseñar a programar al alumno, pues no hay tiempo de hacerlo en un semestre.

Este trabajo parte del hecho de que el lector domina las técnicas de solución de sistemas de ecuaciones de $n \times n$ 
del Álgebra Lineal [10]. De tal manera, se presenta una metodología para resolver un sistema de ecuaciones diferenciales parciales aplicado a un modelo macroscópico del tráfico vehicular. Si bien existen muchos modelos macroscópicos de tráfico [11-14], se eligió el modelo de Greenberg -que fue uno de los primeros- por su sencillez.

Ante el problema de la heterogeneidad de los conocimientos en cómputo de los alumnos, se propone usar un programa de cómputo versátil para resolver numéricamente el problema planteado. El programa propuesto no es complicado de aprender y los resultados pueden graficarse directamente (no es como en los lenguajes tipo $\mathrm{C} / \mathrm{C}++$, Fortran o Java, donde se generan los datos, se guardan en un archivo y luego se utilizar un programa diferente tipo GNUPlot para graficar). La herramienta computacional propuesta es Scilab [15], un programa de libre distribución y que puede instalarse en cualquier sistema operativo: Windows, Linux y Mac.

El modelo de Greenberg [16-18] está basado en las ecuaciones fundamentales de la mecánica de fluidos, suponiendo que el flujo vehicular es un flujo compresible. Greenberg propone que la velocidad es una función de la densidad vehicular, lo cual simplifica las ecuaciones y se puede encontrar una solución analítica. Los datos de Greenberg coinciden perfectamente con su modelo porque fueron tomados en un túnel, para atravesar el río Hudson, que divide a Nueva Jersey de Nueva York.

Para aplicar la metodología didáctica, además de la simulación numérica, en este trabajo se propone realizar una práctica de campo para medir las variables más representativas del tráfico vehicular (velocidad promedio, densidad vehicular y aforo) en una avenida de la Ciudad de México, con el objetivo de obtener condiciones iniciales y de frontera reales para realizar las simulaciones numéricas. Para esto se toman datos en la Ciudad de México, a horas pico, durante aproximadamente 3 horas y en un día laboral. El punto de medición es la carretera libre México-Toluca, a la altura del centro comercial Santa $\mathrm{Fe}$, en la alcaldía Cuajimalpa.

El modelo se resuelve de manera completa (es decir, no se impone la suposición de Greenberg de considerar a la velocidad como una función de la densidad vehicular), usando un método numérico. Se utilizan los datos medidos interpolados como condiciones iniciales y de frontera (condiciones de Dirichlet [19-21]). Los resultados numéricos de la ecuación de Greenberg original, es decir, que toman la ecuación de continuidad homogénea, son inconsistentes con la realidad, pues para la velocidad se obtienen valores negativos y más altos en magnitud que la velocidad promedio del flujo vehicular y la densidad siempre es constante. Los alumnos deben descubrir el porqué de esta inconsistencia, detectar en dónde está el error y proceder a corregirlo. En este caso, suponer la conservación de la masa (ecuación de continuidad homogénea) lleva al error mencionado en el párrafo anterior y se corrige cuando se introduce una función distinta de cero en la ecuación de continuidad [22].
El trabajo está organizado como sigue. En la Sec. 2 se presenta de manera general el modelo de tráfico de Greenberg [16]. El trabajo de campo y un análisis de incertidumbres se describen en la Sec. 3 y los resultados numéricos se exponen en la Sec. 4; además, se señala una comparación con el modelo modificado utilizando el término de la viscosidad. En esta misma sección se muestra la estabilidad y convergencia del método numérico utilizado. Finalmente, la discusión y las conclusiones se muestran en la Sec. 5.

\section{Modelo del Tráfico}

Si se desprecia el término viscoso y de fuerzas externas de las ecuaciones de Navier-Stokes y de continuidad para un flujo compresible [23] en una dimensión espacial, el modelo de Greenberg [16] es:

$$
\begin{aligned}
\frac{\partial u}{\partial t}+u \frac{\partial u}{\partial x} & =-\frac{c^{2}}{k} \frac{\partial k}{\partial x}, \\
\frac{\partial k}{\partial t}+k \frac{\partial u}{\partial x}+u \frac{\partial k}{\partial x} & =0,
\end{aligned}
$$

donde $u(x, t)$ es la velocidad del tráfico $(\mathrm{km} / \mathrm{hr}), k(x, t)$ es la densidad del tráfico $(\mathrm{veh} / \mathrm{km})$ y $c$ es un parámetro que se determina con el flujo de autos.

Es importante mencionar una variable más; el gasto o caudal, que en el sistema del tráfico vehicular se denomina aforo vehicular, $q(x, t)$, con unidades de veh/hr, y se define como:

$$
q(x, t)=u(x, t) k(x, t) .
$$

La Ec. (3) se conoce como la relación fundamental [28] y todas estas variables son funciones tanto de la posición $x$ como del tiempo $t$.

El miembro izquierdo de la Ec. (1) representa la derivada material o total (cuya definición es $D / D t=\partial / \partial t+u \partial / \partial x$ $[23,24])$ de la velocidad $u$. El primer término es conocido como aceleración local y el segundo corresponde a la aceleración convectiva [24]. Este término representa los cambios en la velocidad $u$ en un segmento de longitud $\partial x$, debido a vehículos que se incorporan con velocidad distinta. El miembro derecho de la Ec. (1) es el término de gradiente de presión. Representa cambios de la velocidad por la anticipación de los conductores en condiciones de tráfico a las cuales se enfrentarán posteriormente [25]. Greenberg [16] propuso que la presión vehicular $P$ fuera directamente proporcional a la densidad $k$, de tal manera que $P(k)=c^{2} k$. Esta suposición viene de considerar el flujo vehicular como un gas ideal [22].

La Ec. (2) es la ecuación de continuidad, que representa la conservación de la masa. El hecho de estar igualada a cero indica que no hay adición o pérdida de masa vehicular en el sistema. El primer término del miembro izquierdo es el 


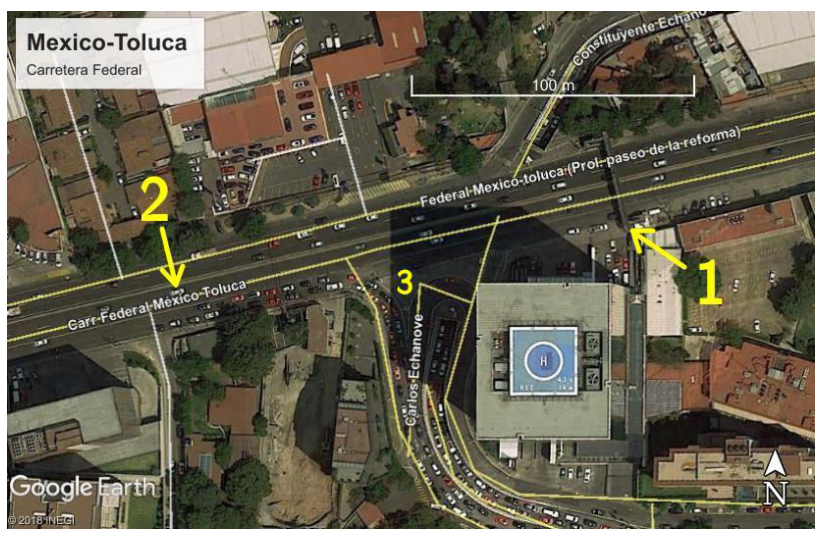

FIgURA 1. Lugar de medición de las variables $u, k$ y $q$. El sentido de circulación es de poniente a oriente, es decir, entrando a la Ciudad de México. Los observadores se colocaron en el punto 1 (puente peatonal) y tomaron como referencia el punto 2 (letrero informativo). Tomado y modificado de GoogleMaps ${ }^{\circledR}$.

cambio de la densidad con respecto al tiempo. El segundo y tercer términos de la Ec. (2) corresponden al gradiente del aforo $\partial q / \partial x$, el cual se desglosa al utilizar la regla de la cadena, ver Ec. (3).

\section{Trabajo de campo}

La toma de datos se realizó en la carretera México-Toluca, solo en el sentido que entra a la Ciudad de México en un segmento de carretera limitado por dos puntos ( 1 un puente peatonal y $\mathbf{2}$ un letrero informativo) que distan entre sí por una distancia aproximada de $150 \mathrm{~m}$ y que abarca una salida de vehículos (ver Fig. 1). Se escogió este tramo por dos razones: 1) es una de las entradas más importantes a la Ciudad de México; 2) contiene una salida intermedia de vehículos; por lo tanto, la ecuación de continuidad Ec. (2) debe modificarse, pues ya no debe igualarse a cero, sino que existe una pérdida de masa vehicular debido a esa salida intermedia. Esta ecuación se reescribe como:

$$
\frac{\partial k}{\partial t}+\frac{\partial q}{\partial x}=\frac{\partial k}{\partial t}+k \frac{\partial u}{\partial x}+u \frac{\partial k}{\partial x}=f(t) .
$$

El valor de la función $f(t)$ se calcula utilizando la forma discreta de la ecuación de continuidad en términos del aforo $q$ :

$$
f(t) \approx \frac{\Delta k}{\Delta t}+\frac{\Delta q}{\Delta x}
$$

donde $\Delta k=k_{1}-k_{2}$ y $\Delta q=q_{1}-q_{2}$, pues la entrada de vehículos es el punto 2 .

Varios observadores se colocaron en el puente peatonal y desde las 7:00 hrs hasta las 10:00 hrs tomaron medidas de aforo, velocidad y fotografías para estimar la densidad vehicular (ver Fig. 2). Esto último se realiza en el trabajo de gabinete.

\subsection{Metodología}

El aforo $q$ se midió de la siguiente manera: en el puente peatonal se hizo el conteo de automóviles durante un minu-

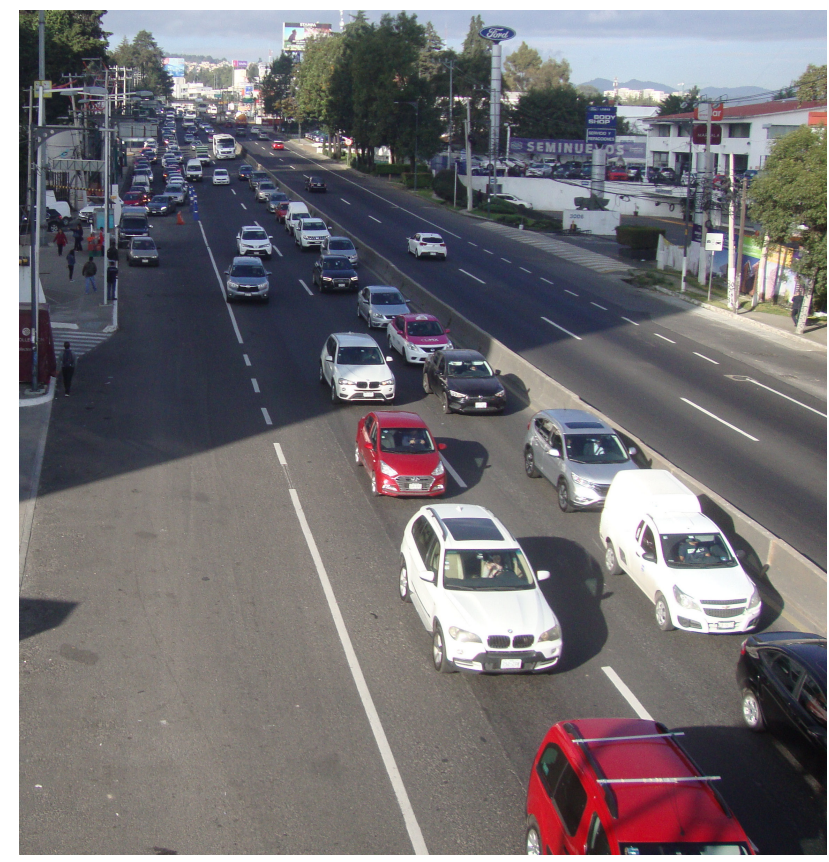

FIGURA 2. Fotografía para estimar la densidad vehicular, tomada el 13 de noviembre de 2018, a las 8:32 hrs. Foto: P. E. Olivera.

to, en intervalos de 10 minutos; es decir, a las 7:00 hrs y durante un minuto (hasta las 7:01 hrs) se contaron los vehículos que pasaban por ambos puntos (unos observadores midieron los que pasaban por el soporte para letrero y otros observadores hicieron el conteo de los autos que circulaban por debajo del puente). El mismo proceso se realizó a las 7:10 hrs, a las 7:20 hrs, etc. Así, se registró el aforo en el punto inicial y en el punto final del segmento de carretera estudiado.

La velocidad promedio de los vehículos se estimó con el procedimiento siguiente. Al tomar un punto de referencia, como un letrero o un semáforo a cierta distancia del puente, se contó el tiempo que le tomaba a algún vehículo arbitrario con recorrer esa distancia. Esto se hizo después de medir el aforo, es decir, a las 7:01 hrs, a las 7:11 hrs, etc. Después, en trabajo de gabinete, se midió la distancia desde el puente peatonal a la referencia con la herramienta Google Earth Pro ${ }^{\circledR}$ [26]. Con esas cantidades se estimó la velocidad promedio a la que iban los vehículos a la hora de medición.

Se utilizó la misma metodología para estimar la distancia para el cálculo de la densidad vehicular y se tomaron fotografías después de hacer la medición del tiempo para la velocidad, es decir, a las 7:02 hrs, a las 7:12 hrs, etc. Por ejemplo, en la fotografía de la Fig. 2, que se tomó a las 8:32 hrs, se cuentan aproximadamente 75 vehículos en la carretera, de los cuales más de la mitad $(\approx 41)$ están dejando la vialidad. El punto 2 se encuentra a una distancia de $\approx 150 \mathrm{~m}$ del punto $\mathbf{1}$, de manera que, en ese instante, la densidad medida es de $500 \mathrm{veh} / \mathrm{km}$. Cabe mencionar que la vialidad tiene $4 \mathrm{ca}-$ rriles, por tanto, si se asume que los vehículos tienen un largo promedio de $5 \mathrm{~m}$ y existe una separación entre vehículos de 
0.5 a $1 \mathrm{~m}$ cuando hay embotellamiento, se tiene una capacidad de 667 vehículos por kilómetro. En el segmento estudiado se tendrán un máximo de 100 vehículos.

\subsection{Análisis de incertidumbres}

Debido a que las escalas espacial y temporal a las cuales se tomaron las mediciones fueron metros y segundos, se definieron las incertidumbres para el espacio y el tiempo como $\delta x= \pm 1 \mathrm{~m}=1 \times 10^{-3} \mathrm{~km}$ y $\delta t= \pm 1 \mathrm{~s}=2.78 \times 10^{-4} \mathrm{hr}$, respectivamente. La propagación de incertidumbres se calculó siguiendo el procedimiento de Oda [27]. Para la velocidad $u$ el error asociado $\delta u$, se estimó con la fórmula

$$
\delta u=\frac{x_{0} \delta t+t_{0} \delta x}{t_{0}^{2}}
$$

donde $x_{0}=0.15 \mathrm{~km}$ y $t_{0}$ es el tiempo que le tomó a un vehículo (tomado como referencia) recorrer $150 \mathrm{~m}$. Para calcular la incertidumbre asociada al valor máximo registrado de la velocidad, se utiliza el menor valor del tiempo $t_{0}=21.4 \mathrm{~s}$ en $150 \mathrm{~m}$. Esto corresponde a una velocidad de $25 \mathrm{~km} / \mathrm{hr}$; así, se tiene $\delta u=1.32 \mathrm{~km} / \mathrm{hr}$. Al definir el error asociado a la variable medida como la razón de su incertidumbre y la variable misma, multiplicada por $100(\%)$, se tiene que el error para la velocidad máxima medida es de $1.32 / 25=5.3 \%$. Es importante destacar que este es el máximo obtenido para todas las mediciones. El error máximo admitido en este trabajo es de $10 \%$, por tanto los errores de medición obtenidos son aceptables.

Con fórmulas similares a la Ec. (6) se calculan la incertidumbres de la densidad $\delta k$ y del aforo $\delta q$. Para las tres variables, se presentan los errores asociados a la cantidades máxima y mínima medidas de cada variable, es decir, $u_{0} \pm \delta u, k_{0} \pm \delta u$ y $q_{0} \pm \delta q$, en la Tabla I.

Como las mediciones se tomaron cada diez minutos, durante aproximadamente tres horas, se tienen valores temporales discretos para las funciones $u, k$ y $f$. Para estimar la función $f$ se toma $\Delta t=1 / 6 \mathrm{hr}$ y $\Delta x=0.15 \mathrm{~km}$, ver Ec. (5).

Para completar la función en un dominio más continuo, es decir, calculando en los puntos faltantes del dominio temporal de la solución (el lapso temporal de los datos medidos es de $1 / 6 \mathrm{hr}$, mientras que el paso temporal de la solución puede ser más pequeño), se realizó un procedimiento de interpolación utilizando un spline cúbico [30].

TABLA I. Incertidumbres asociadas a los valores máximos y mínimos de las variables medidas en campo.

\begin{tabular}{cc}
\hline Variable & Valores \\
\hline$u$ & $u_{\text {máx }} \pm \delta u=25 \pm 1.32 \mathrm{~km} / \mathrm{hr}$ \\
& $u_{\text {mín }} \pm \delta u=3 \pm 0.037 \mathrm{~km} / \mathrm{hr}$ \\
\hline$k$ & $k_{\text {máx }} \pm \delta k=653 \pm 11 \mathrm{veh} / \mathrm{km}$ \\
$k_{\text {mín }} \pm \delta k=200 \pm 8 \mathrm{veh} / \mathrm{km}$ \\
\hline \multirow{2}{*}{$q$} & $q_{\text {máx }} \pm \delta q=4680 \pm 2 \mathrm{veh} / \mathrm{hr}$ \\
& $q_{\text {mín }} \pm \delta q=2100 \pm 2 \mathrm{veh} / \mathrm{hr}$ \\
\hline
\end{tabular}

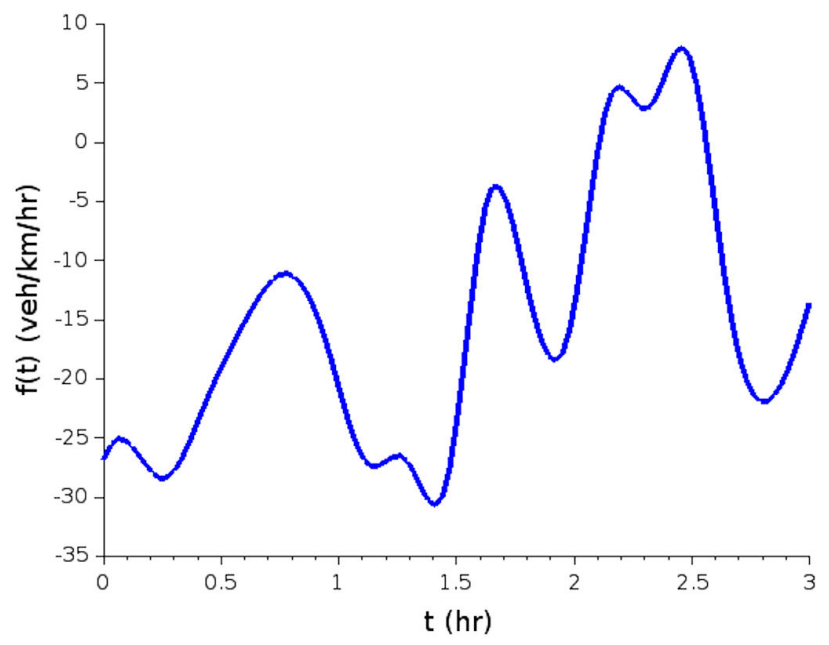

FIGURA 3. Función $f(t)$ de la Ec. (5) con datos interpolados con un spline cúbico [30].

En la Fig. 3 se puede observar la forma de la función $f(t)$ Ec. (5) interpolada. Esta muestra que la función $f(t)$ tiene más valores negativos que positivos. Esto significa que salieron más vehículos de los que entraron. Lo anterior se explica porque hubo una salida de vehículos intermedia en el segmento de vialidad estudiado. En este caso fue la calle Carlos Echanove (salida marcada con el número 3 en la Fig. 1).

\section{Resultados}

Debido a que el modelo de Greenberg requiere conocer un parámetro característico de flujo vehicular, en este caso $c^{2}$ de la Ec. (1), este se estima utilizando el diagrama fundamental del tráfico, que relaciona la velocidad y la densidad [16,28]. En este caso se realizó una regresión por mínimos cuadrados ordinarios, para correlacionar las variables $u$ y $k$ (medidas en la carretera México-Toluca en los puntos señalados), como una función exponencial negativa, de acuerdo con la suposición original de Greenberg [16].

Según los resultados de la regresión, estas dos variables tienen una correlación débil, pues el coeficiente de determinación es $R^{2}=0.27$. El valor calculado para $c$ es de $1 / 0.01882=53.14$ (ver Fig. 4 y Ec. (17) de [16]).

El sistema de Ecs. (1.2) se resolvió numéricamente usando un método de diferencias finitas de segundo orden para las derivadas espaciales y de primer orden para las derivadas temporales, cuyas fórmulas se pueden encontrar en [30-33].

Como condición inicial se utilizó el primer valor medido (es decir, el de las 7:00 am) de las variables en alguno de los puntos de medición. Las condiciones de frontera para la densidad se impusieron como la derivada normal igualada a cero (condiciones de Neumann), que suponen físicamente que el valor de la densidad en la frontera y en el punto adyacente a ella es el mismo. Para la velocidad se establecieron condiciones de Dirichlet, que son los valores $u(0, t)$ y $u(L, t)$, donde 0 representa el punto inicial, por donde entran los vehículos, y $L$ el punto final, por donde salen los vehículos; 


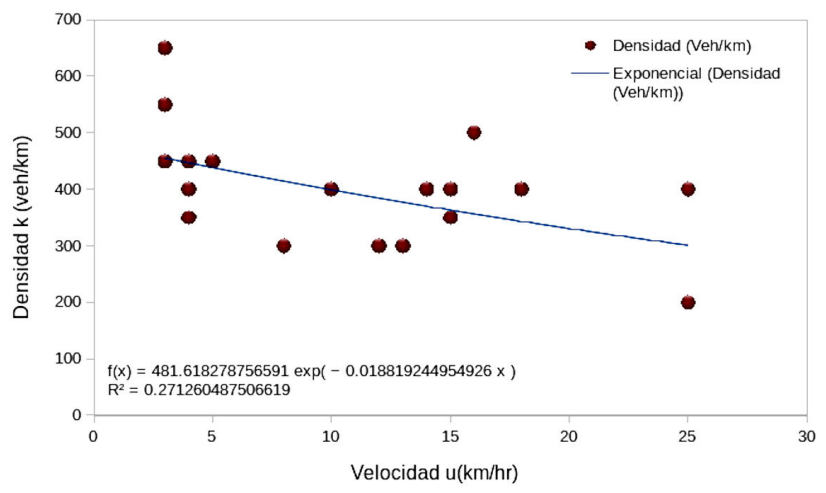

FIGURA 4. Estimación de la densidad en función de la velocidad a partir de datos medidos utilizando un método de regresión.

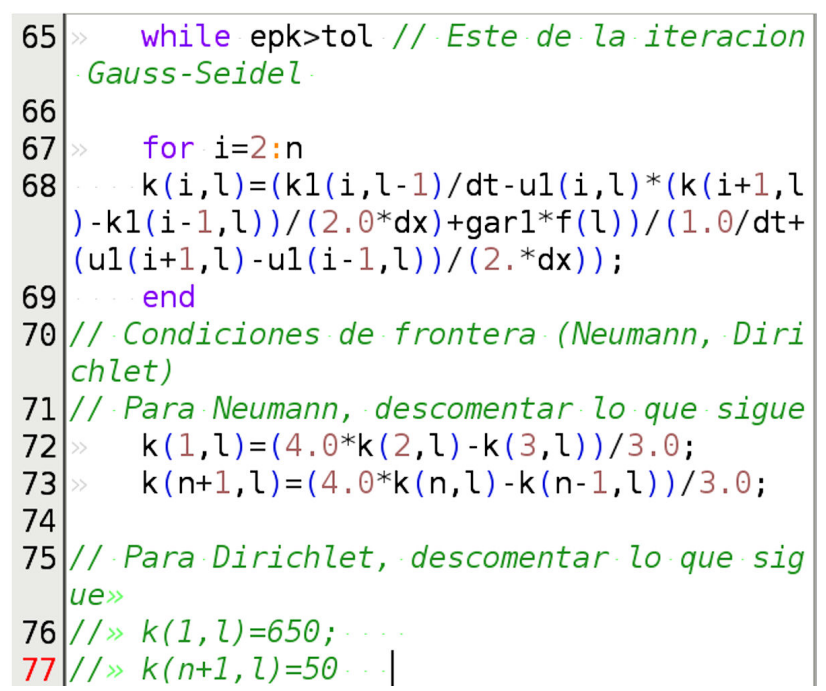

FIGURA 5. Extracto del código numérico para calcular la densidad vehicular $k$ escrito en el editor de textos propio de Scilab [15].

vehículos; es decir, los extremos del segmento de vialidad estudiado.

Para resolver el sistema de ecuaciones generado por el método de diferencias finitas se utilizó un método iterativo (Gauss-Seidel $[30,31]$ ) con una tolerancia tol $=10^{-10}$, que es un valor usado comúnmente en la investigación. Aunque en docencia, a los alumnos se les permiten tolerancias entre $10^{-4}$ y $10^{-5}$.

El método numérico se implementó en un código numérico escrito en Scilab [15], que es un programa versátil de código abierto y gratuito, que se puede instalar en cualquier sistema operativo (Windows, Linux y Mac). En las Figs. 5 y 6 se muestran un par de extractos del código numérico para calcular la densidad $k$ y para obtener la velocidad $u$ escritos en el editor propio de Scilab.

\subsection{Modelo original: $f(t)=0$}

Como se mencionó en la introducción, los datos que Greenberg midió encajan bien en su modelo porque se tomaron en segmentos de vialidad que no tienen entradas y salidas intermedias de vehículos, por lo tanto, la masa vehicular se con-

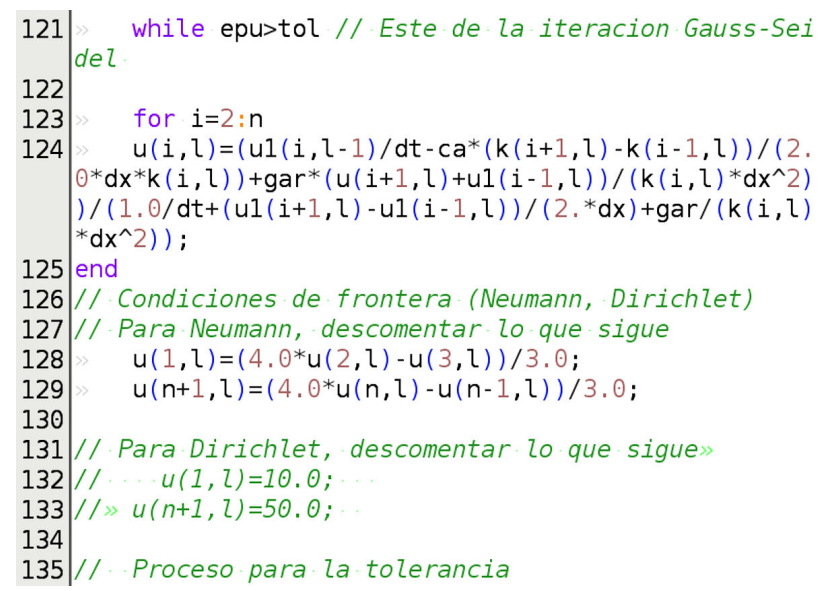

FIGURA 6. Extracto del algoritmo para calcular la velocidad $u$ escrito en el editor de textos propio de Scilab [15].

serva. En este trabajo llamaremos modelo original al sistema (1.2), es decir, cuando $f(t)=0$ en la Ec. (4).

En este caso, se tiene una importante salida intermedia de vehículos, por lo que surge la siguiente cuestión: ¿Qué pasa si se utiliza el modelo original usando los datos medidos como datos de entrada? Con el código implementado se pueden realizar tantos experimentos numéricos como se desee. En particular para contestar esta pregunta se realizó un primer ejercicio que consistió en hacer una simulación con el modelo original de Greenberg.

En las Figs. 7 y 8 se muestran los resultados de la velocidad y de la densidad para el caso del modelo original de Greenberg, resuelto numéricamente. La velocidad como función del tiempo y la posición se presenta en la Fig. 7. Se pueden observar picos, tanto negativos como positivos a lo largo de la vialidad de $1 \mathrm{~km}$ de longitud. En la misma figura se pueden observar las condiciones de frontera como funciones "suavizadas" del tiempo.

A pesar de tener esas funciones "suavizadas" (por interpolación) en la frontera, el modelo hace que la velocidad en un punto cualquiera de la vialidad (por ejemplo, $x=0.5 \mathrm{~km}$ ) permanezca constante prácticamente todo el tiempo (salvo en

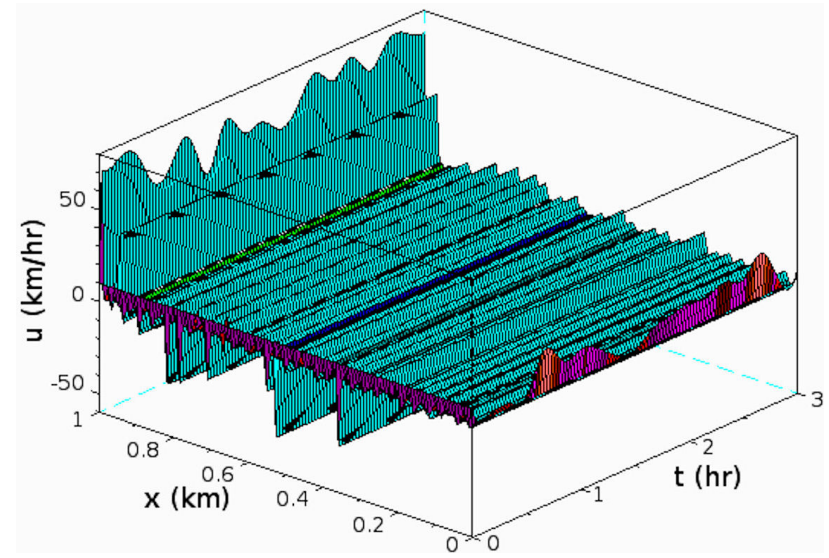

FIGURA 7. Velocidad $u(\mathrm{~km} / \mathrm{hr})$ calculada con el modelo original de Greenberg. Los colores son asignados por defecto por el graficador de Scilab. 


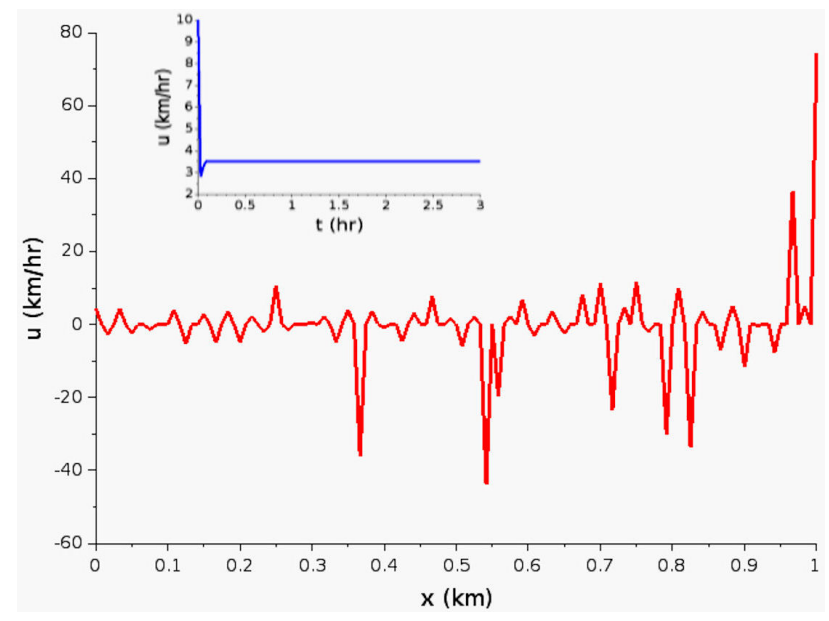

FIGURA 8. Velocidad $u(\mathrm{~km} / \mathrm{hr})$ a lo largo de todo el dominio espacial en un tiempo fijo. En una subgráfica se muestra la velocidad vehicular $u(\mathrm{~km} / \mathrm{hr})$ en un punto de la vialidad durante todo el tiempo para el modelo original de Greenberg.

tiempos tempranos, como puede observarse en la subgráfica de la Fig. 8). Se eligió un punto particular para mostrar en la gráfica, donde la velocidad se mantiene en $3.5 \mathrm{~km} / \mathrm{hr}$. Sin embargo, existen puntos donde se mantiene constante en cero o incluso en valores negativos. No es posible que un automóvil se mantenga en reversa a más de $40 \mathrm{~km} / \mathrm{hr}$ durante 3 horas en una vialidad congestionada.

La gráfica de la velocidad a un tiempo fijo ( $t=1.5 \mathrm{hr})$ se muestra en la Fig. 8. La velocidad en función de la posición $x$ presenta fluctuaciones que van entre los $-50 \mathrm{~km} / \mathrm{hr}$ y los $40 \mathrm{~km} / \mathrm{hr}$. Salvo en las cercanías de $x=1 \mathrm{~km}$, las velocidades positivas no sobrepasan los $10 \mathrm{~km} / \mathrm{hr}$. Las velocidades negativas pueden representar a vehículos que van en reversa, aunque $50 \mathrm{~km} / \mathrm{hr}$ en reversa es una vialidad congestionada es muy poco probable. El indicio de que la magnitud de las velocidades negativas sea, en general, mayor que las velocidades positivas sugiere que se debe ajustar el modelo.

Por otro lado, la densidad mostrada en la Fig. 9 se mantiene constante en todo tiempo. Este resultado es muy

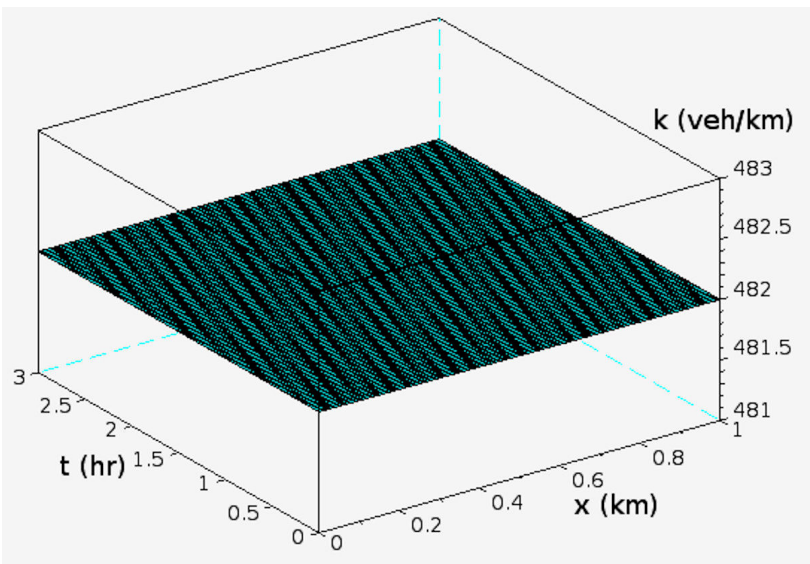

FIGURA 9. Densidad vehicular $k$ (veh/km) calculada con el modelo original de Greenberg. Los colores se asignan por defecto por el graficador de Scilab. poco realista. El obtener una densidad constante a todo tiempo y velocidades negativas, indica que el modelo original (1. 2) ofrece resultados no muy satisfactorios.

\subsection{Modelo modificado $f(t) \neq 0$}

Los resultados anteriores sugieren que se debe usar la modificación a la ecuación de continuidad del modelo original de Greenberg, de tal manera que se considere la pérdida o ganancia de masa vehicular en un segmento de vialidad con entradas y salidas intermedias. Lo llamaremos modelo modificado si $f(t) \neq 0$ en la Ec. (4).

Al utilizar la función expuesta en la Fig. 3 para $f(t)$, los resultados numéricos de velocidad y densidad para el modelo modificado de Greenberg se muestran en las Figs. 10 y 11, respectivamente. La velocidad sigue presentando valores negativos, pero esos máximos no rebasan los $-30 \mathrm{~km} / \mathrm{hr}$. Además, los máximos positivos no van más allá de $60 \mathrm{~km} / \mathrm{hr}$, que es un valor aceptable en esta situación.

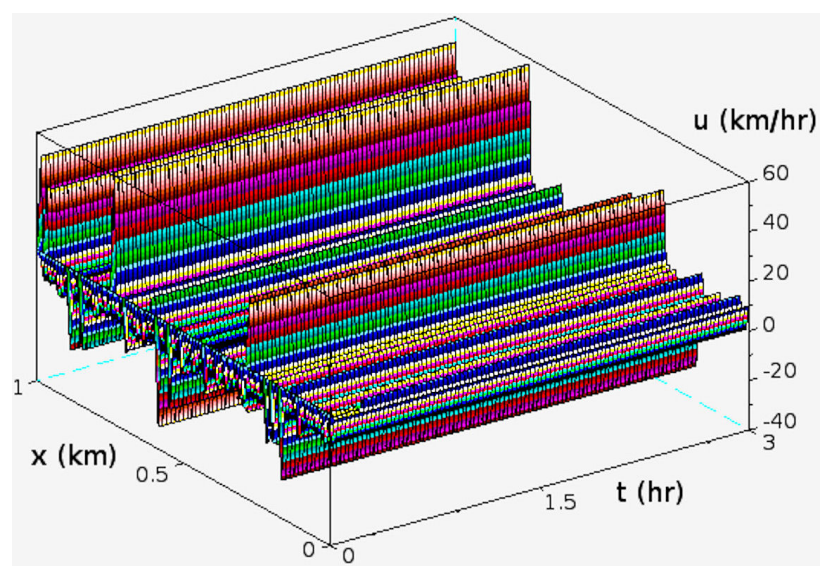

FIGURA 10. Velocidad vehicular promedio ( $\mathrm{km} / \mathrm{hr}$ ) para todo tiempo usando el modelo modificado de Greenberg. Los colores se asignan por defecto por el graficador de Scilab.

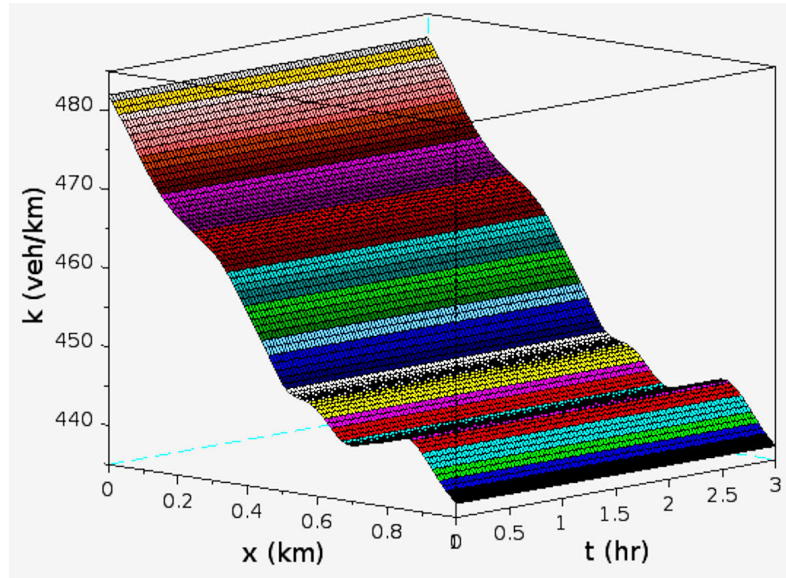

FIGURA 11. Densidad vehicular media (veh/km) para todo tiempo para el modelo modificado de Greenberg. Los colores se asignan por defecto por el graficador de Scilab. En las gráficas de las siguientes secciones, los colores son asignados por el usuario, al utilizar algunas sencillas instrucciones o el editor de gráficos propio de Scilab. 
El modelo modificado presenta resultados un poco más realistas que el modelo original, pues al saberse que el límite en esta vialidad es de $80 \mathrm{~km} / \mathrm{hr}$, los datos simulados presentan resultados que no lo sobrepasan aún sin haber puesto ninguna restricción al respecto. Además, considerar entradas y salidas intermedias de vehículos $(f(t) \neq 0)$ dio picos de velocidades positivas más grandes que $10 \mathrm{~km} / \mathrm{hr}$ y redujo la magnitud de las velocidades negativas, pero no las eliminó.

Por otra parte, la densidad ya no representa una constante $\mathrm{y}$, para este caso específico, tiende a disminuir en el lapso durante el cual se tomaron las mediciones (ver Fig. 11).

Se puede observar que, al considerar el término $f(t)$ diferente de cero, se obtienen resultados más aceptables físicamente que si $f(t)=0$, aunque, permanece la inquietud sobre las velocidades negativas relativamente altas.

Los modelos en varias áreas de estudio arrojan valores negativos de las variables que estudian, sin que tengan algún significado (por ejemplo, en Epidemiología, no tiene sentido obtener valores negativos del número de individuos infectados, o en Economía, los precios negativos o las cantidades producidas negativas carecen de sentido económico). Así que los estudiosos simplemente no toman en cuenta esos valores negativos.

En este punto es necesario plantear al alumno si es posible dar un argumento físico para tomar el valor absoluto de la velocidad, despreciar los valores negativos que físicamente indicarían vehículos en reversa o mejorar el modelo.

\subsection{Viscosidad en el modelo modificado}

Los estudiantes que tengan curiosidad propondrán mejorar el modelo. Los modelos se construyen de acuerdo con las necesidades del modelador. En este caso, una forma de mejorar el modelo es considerar cada vez más términos en la ecuación dinámica, por ejemplo, sumar el término viscoso en el segundo miembro de la Ec. (1). Según Treiber y Kesting [34], el término disipativo mejora las propiedades numéricas del modelo, para eliminar las ondas de choque e investigar los efectos de la difusión numérica que son inevitables cuando se integran numéricamente modelos macroscópicos.

Así, el término viscoso puede aproximarse con diferencias finitas como

$$
\frac{\eta}{k} \frac{\partial^{2} u}{\partial x^{2}} \approx \frac{\eta}{k(i, j)} \frac{u(i+1, j)-2 u(i, j)+u(i, j-1)}{\Delta x^{2}},
$$

de acuerdo con las fórmulas deducidas de la serie de Taylor a segundo orden [30,31]. El código numérico mostrado en la Fig. 6 ya contempla el término viscoso, en el cual la variable gar sirve como un interruptor: si gar $=0$, se resuelve el caso no viscoso, en otro caso, gar $=\eta$ en la Ec. 7 representa la viscosidad. Al realizar un análisis dimensional, $\eta$ debe tener unidades de número de vehículos por velocidad (veh $\mathrm{km} / \mathrm{hr}$ ).

En modelos de tráfico vehicular, la viscosidad es interpretada como la anticipación del conductor de alto grado [25]. De acuerdo con esta interpretación y con las unidades que tiene $\eta$, se puede definir la viscosidad como la distancia $\delta_{v}$ que el conductor alcanza a ver (para anticiparse a cualquier eventualidad) y el tiempo $\tau_{a}$ que ocupa en realizar la acción de anticipación. Como referencia se toma el valor utilizado por Delgado et al. [22], que es $\eta=600 \mathrm{~km} / \mathrm{hr}$. Se puede elegir cualquier combinación de $\delta_{v} \mathrm{y} \tau_{a}$ para obtener los valores de viscosidad deseados. Por ejemplo, para $\eta=600 \mathrm{~km} / \mathrm{hr}$ se pueden tomar $\delta_{v}=167 \mathrm{~m} \mathrm{y} \tau_{a}=1 \mathrm{~s}$.

Ahora se tiene una ecuación de segundo orden en la coordenada espacial y de primer orden en la coordenada temporal, por lo tanto se requiere una condición inicial y dos condiciones de frontera. Para ambas variables, $u \mathrm{y} k$, se efectuaron mediciones en las fronteras, es decir, en los puntos $\mathbf{1}$ y $\mathbf{2}$ de la Fig. 1. Las simulaciones se realizaron imponiendo condiciones de frontera de Dirichlet y de Neumann para ambas ecuaciones. Los mejores resultados se obtienen imponiendo condiciones de Dirichlet para la velocidad y de Neumann para la densidad.

Se llevan a cabo simulaciones usando diferentes valores de viscosidad y se comparan con el caso invíscido $(\eta=0)$ y

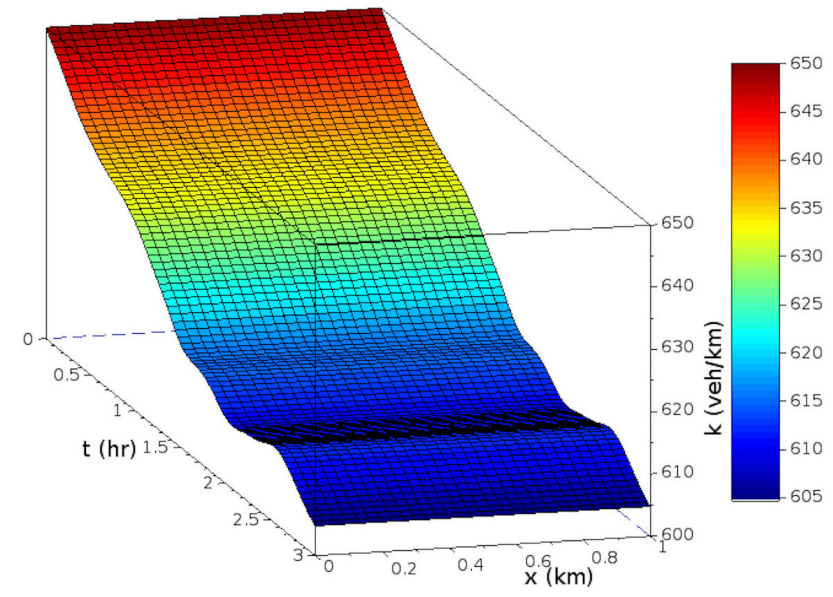

FIGURA 12. Densidad en el caso del modelo modificado con viscosidad para $\eta=600$.

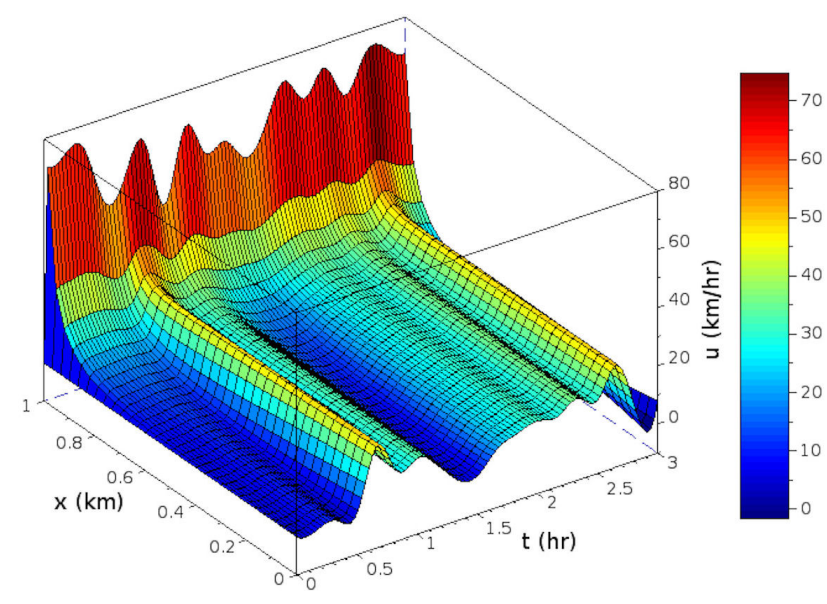

FIGURA 13. Velocidad en el caso del modelo modificado con viscosidad para $\eta=600$. 


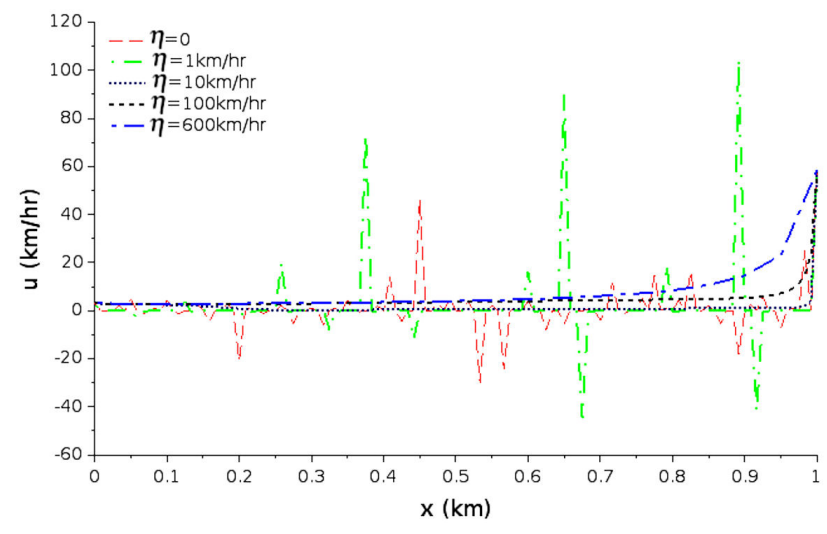

FIGURA 14. Velocidad como función de la posición a un tiempo fijo: $u(x, 1.5)$.

el caso viscoso de referencia ( $\eta=600 \mathrm{~km} / \mathrm{hr}$ ). El conjunto $A_{\eta}$ de valores de viscosidad utilizados para realizar los cálculos es:

$$
A_{\eta}=\{0,1,10,100,600\}
$$

Las gráficas de la densidad y la velocidad para $\eta=600$ se muestran en las Figs. 12 y 13.

En este caso, la densidad (Fig. 12) tiene un comportamiento parecido al caso no viscoso (ver Fig. 11). Esto se debe a que la ecuación para la densidad Ec. (4) no cambió, por tanto las variaciones en la solución parecen ser muy similares en su forma (no en su magnitud) a simple vista, aunque no lo son. La tendencia es a decrecer conforme pasa el tiempo, pero este comportamiento solo es válido para las 3 $\mathrm{hr}$ durante las cuales se midieron los datos. Es muy probable que la tendencia cambie a lo largo del día.

En el caso de la velocidad (Fig. 13), los resultados muestran un comportamiento muy diferente al del caso no viscoso: en primer lugar, hay un cambio de la velocidad con respecto al tiempo, que no ocurría en el caso no viscoso. En segundo lugar, los valores negativos de la velocidad van desapareciendo conforme el valor de la viscosidad. Finalmente no se observan "picos" como en el caso no viscoso, sino que la gráfica es suave. Esto motiva a estudiar el comportamiento cuando hay un cambio gradual en los valores de la viscosidad.

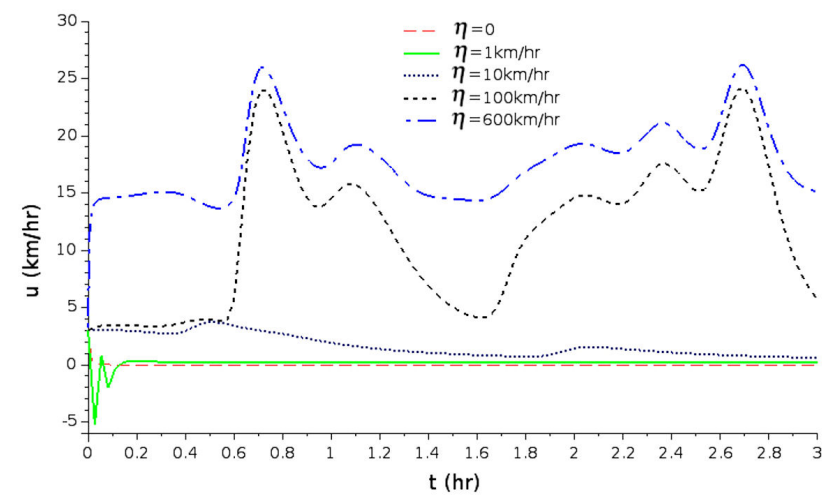

FIGURA 15. Velocidad como función del tiempo a una posición fija: $u(0.78, t)$.
Las Figs. 14 y 15 muestran gráficas de la velocidad contra la posición y el tiempo, respectivamente. La velocidad contra la posición se muestra en la Fig. 14 a un tiempo fijo $t=1.5 \mathrm{hr}$.

El caso $\eta=1 \mathrm{~km} / \mathrm{hr}$ presenta picos de velocidades positivas y negativas con magnitud mayor al caso no viscoso. Pero al aumentar la viscosidad (a partir de $\eta=10 \mathrm{~km} / \mathrm{hr}$ ), los picos desaparecen y la velocidad aumenta de un extremo a otro de la vialidad. La magnitud de la velocidad aumenta con la magnitud de la viscosidad.

La velocidad contra el tiempo se presenta en la Fig. 15 a una posición fija $x=780 \mathrm{~m}$. El caso invíscido muestra una velocidad constante $u(0.78, t)=0$ (salvo para los primeros tiempos, como se vio anteriormente). El caso de $\eta=1 \mathrm{~km} / \mathrm{hr}$ se comporta de manera similar al caso invíscido: es constante salvo en los primeros tiempos, pero ahora es ligeramente mayor que cero. En el caso de $\eta=10 \mathrm{~km} / \mathrm{hr}$; la velocidad tiene dos pequeños máximos a la media hora y luego de dos horas. Para los casos de viscosidad $\eta \geq 100, \mathrm{~km} / \mathrm{hr}$ la velocidad cambia con el tiempo.

En la literatura se encuentra que la densidad y la velocidad tienen una relación inversa $[12,16,28]$ : cuando la densidad vehicular es pequeña, los vehículos pueden ir prácticamente a la velocidad que quieran, y preferirán ir al límite de velocidad o, en muchos casos, lo superaran. Pero cuando la densidad es grande, suponiendo un embotellamiento, la velocidad es cero, pues los vehículos no pueden avanzar porque tienen el obstáculo de los autos de enfrente y de atrás. Es decir, si la densidad es baja, la velocidad depende de las condiciones del camino. Por otro lado, si la densidad es alta, la velocidad dependerá de las condiciones del flujo.

En la Fig. 16 se presenta el espacio fase, también conocido como relación velocidad - densidad del diagrama fundamental [28]. En este caso la velocidad se relaciona con la densidad obtenidas numéricamente. A simple vista se puede observar que esta relación es directa, es decir, la pendiente global de las curvas mostradas es positiva, lo que contradice la teoría del tráfico convencional. No obstante, existe un par de detalles a considerar. El dominio de la densidad mostrado en la Fig. 16 es un pequeño segmento del dominio completo: $k \in[1,667] \mathrm{veh} / \mathrm{km}$. Este segmento se encuentra muy

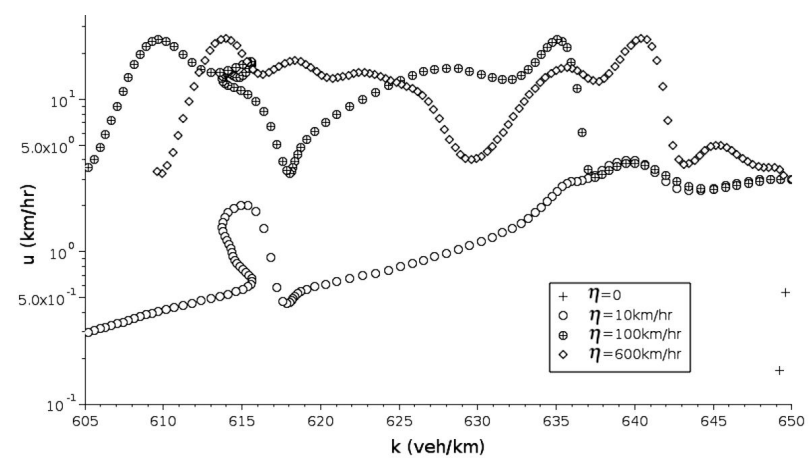

FIGURA 16. Espacio fase para 4 casos del modelo de Greenberg viscoso. 
cercano al límite superior, es decir, al valor de embotellamiento. La velocidad tiene valores entre los $0.3 \mathrm{~km} / \mathrm{hr}$ y los $30 \mathrm{~km} / \mathrm{hr}$ aproximadamente, que no corresponde ni a la mitad del límite permitido para esa vialidad.

Por otro lado, la velocidad es más alta si la densidad $\eta$ es más grande, lo que parece contradecir la teoría de la mecánica de fluidos, pues la viscosidad es una especie de fuerza de fricción y se opone al movimiento. Esto se debe plantear a los alumnos para que argumenten una explicación física (en este trabajo, se presenta en las discusiones).

\subsection{Estabilidad y convergencia del método numérico}

\subsubsection{Estabilidad}

La estabilidad del método se prueba usando el número de Courant-Friedrichs-Lewy [22] o número de Courant, el cual está definido como [35]:

$$
C o=\frac{|u| \Delta t}{\Delta x}
$$

El método es estable si $C o<1$, pues esto indica que una partícula con velocidad $|u|$ no se saldrá de la celda de tamaño $\Delta x$.

La Fig. 17 muestra el número de Courant máximo y el número de Courant promedio para el caso del modelo modificado con viscosidad $\eta=600 \mathrm{~km} / \mathrm{hr}$. Como se puede observar, el número de Courant máximo tiene valores menores a 0.9 y el número de Courant promedio está muy por debajo del número de Courant máximo. Cabe mencionar que los números de Courant para los demás casos tienen comportamientos diferentes que el mostrado en la Fig. 17, pero no sobrepasan el valor de 1 , salvo en los casos de $\eta=0$ y $\eta=1$ donde se encontraron dos picos que llegaron a $C o_{\text {máx }}=1.2$, pero después los valores fueron menores que 1. Esto significa que se puede considerar que el método es estable numéricamente.

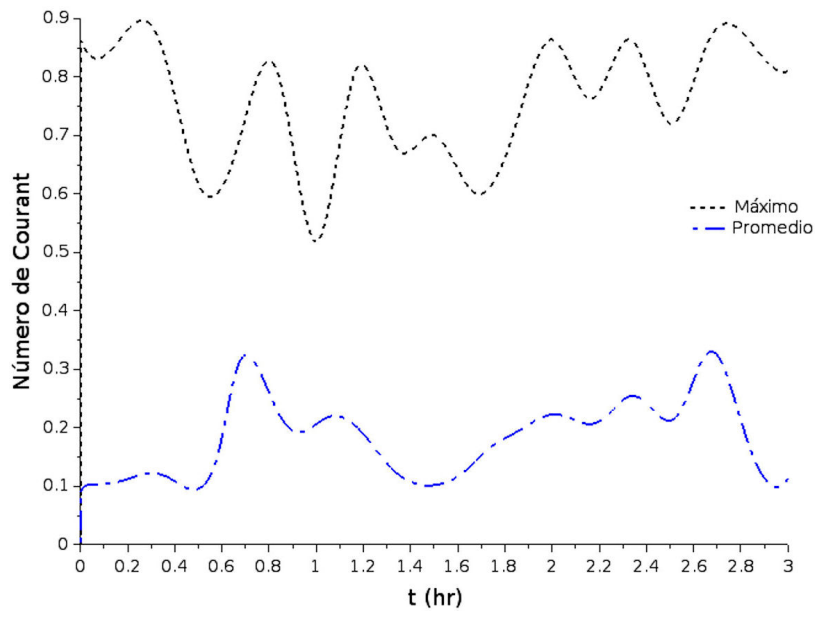

FIGURA 17. Número de Courant, máximo y promedio, para el caso del modelo viscoso con $\eta=600 \mathrm{~km} / \mathrm{hr}$.
TABLA II. Número de puntos $n$ y $m$ y el número de veces que se refinó la malla de puntos.

\begin{tabular}{ccc}
\hline Refinamiento & $n($ para $x)$ & $m($ para $t)$ \\
\hline (1) & 16 & 201 \\
(2) & 31 & 901 \\
(3) & 61 & 2501 \\
(4) & 71 & 7501 \\
\hline
\end{tabular}

\subsubsection{Convergencia}

Una forma de probar la convergencia del método es realizar un refinamiento de la malla de puntos en donde se calcula la solución. Si $n$ es el número de puntos en $x$ y $m$ es el número de puntos en $t$, el refinamiento de la malla se realiza aumentando $n$ y $m$ cada vez que se calculan las soluciones. Se dice que el método converge si los valores de las variables confluyen en la solución del sistema conforme se refina la malla de puntos. En la Tabla II se muestra cómo fue el refinamiento de la malla en este caso.

Las Figs. 18 y 19 muestran un acercamiento de las gráficas de velocidad $u$ y densidad $k$, respectivamente, para el caso con viscosidad $\eta=600 \mathrm{~km} / \mathrm{hr}$. En la Fig. 18 se puede observar que únicamente el caso de 201 puntos es diferente a los demás; las gráficas para los casos de $m=2501$ y $m=7501$ están prácticamente encimadas.

En el caso de la densidad, se muestra un acercamiento de las gráficas de la densidad calculada con diferente número de puntos en los dominios espacial y temporal (en la Fig. 19 solo se presenta el número de puntos en el dominio temporal, el correspondiente al dominio espacial se puede consultar en la Tabla II).

En este caso sí se observa una diferencia entre las distintas gráficas, sobre todo en los primeros tiempos. Sin embargo, se puede observar cómo el caso de $m=2501$ ya es muy cercano al caso de $m=7501$. Cabe mencionar que se hizo una prueba con $n=65$ y $m=3501$ (que no se presenta en la Tabla II), pero no se graficó con las demás porque se encima con la curva de $m=7501$. Así, se puede apreciar mejor

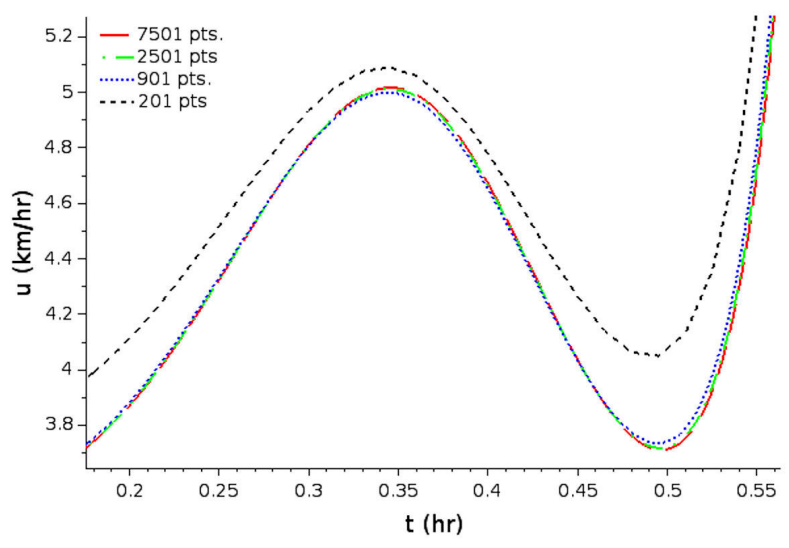

FIGURA 18. Convergencia del método para $u$ 


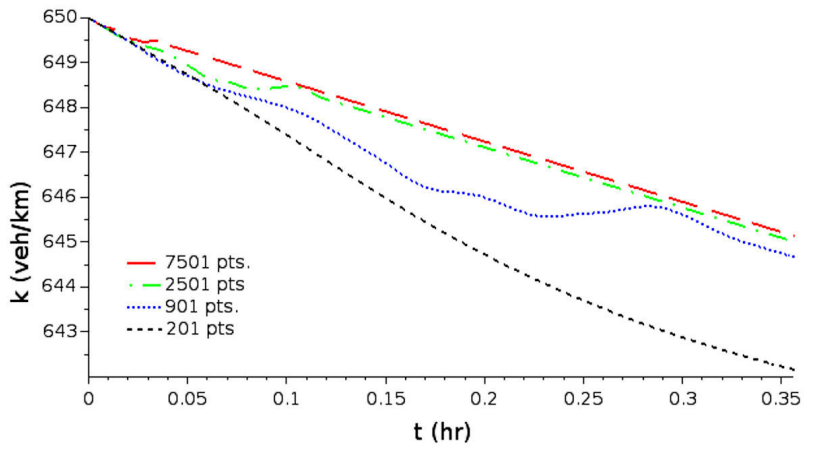

FIGURA 19. Convergencia del método para $k$.

cómo, conforme se aumenta el número de puntos, la solución numérica va convergiendo a la solución del sistema de ecuaciones.

\subsubsection{Tiempo de ejecución}

Para definir si el ejercicio es adecuado para realizarse en un salón de clases, se midió el tiempo de ejecución en el cálculo de la solución.

En la Fig. 20 se muestra que el tiempo de ejecución es directamente proporcional al número de puntos, que en este caso se encuentra en el eje de las absisas representado como $n \times m$.

El número máximo en este ejercicio fue de $71 \times 7,501=$ 532,571 puntos y la gráfica muestra que Scilab tardó poco menos de 1 hora en realizar el cálculo, incluyendo las gráficas en bruto, es decir, sin el nombre de los ejes, cambio en el grosor y color de las líneas, cambio en el tamaño de la fuente, etc.

Los cálculos se realizaron en una computadora laptop HP, con un procesador intel Core i3 de 8 va generación a $2.1 \mathrm{GHz}$, con unidad de disco de estado sólido de $256 \mathrm{~Gb}$, y memoria SDRAM DDR4 de $8192 \mathrm{Mb}$. Esta es una computadora que un alumno de ciencias o ingeniería utilizaría como equipo personal.

El tiempo máximo de cálculo es de aproximadamente $1 \mathrm{hr}$ y una clase de Análisis Numérico o Física Computacional os-

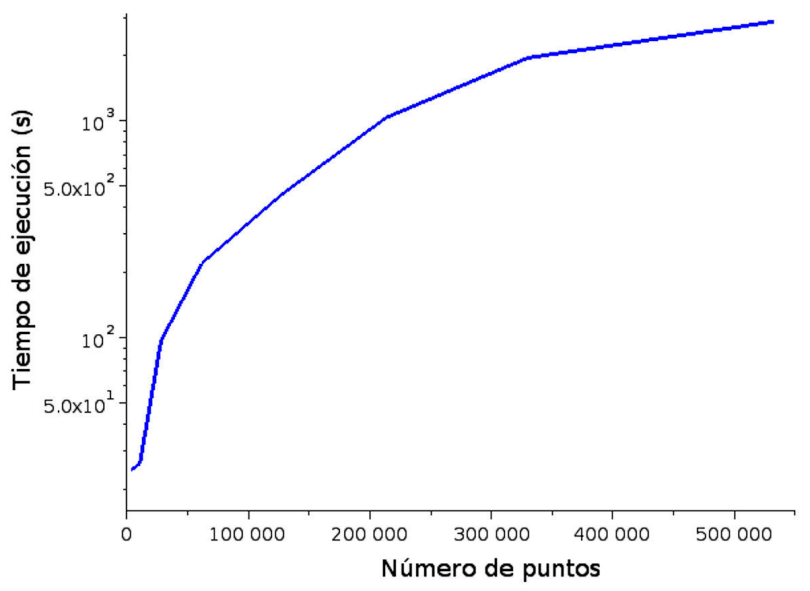

FIGURA 20. Tiempo de ejecución respecto al número de puntos. cila entre 2 y 3 hrs, así que en la clase puede ejecutarse sin problema hasta el caso $n=31$ y $m=901$, el cual tarda alrededor de minuto y medio (pensando que las computadoras que existen en una sala de cómputo de una facultad son mucho menos poderosas que la utilizada para hacer este ejercicio y este caso podría ejecutarse hasta en $5 \mathrm{~min}$ ).

Los casos más tardados se dejan como ejercicio para casa, en los cuales prácticamente lo único por hacer es cambiar el número de puntos en el código.

\section{Discusión y conclusiones}

Se plantea una metodología de resolución de ecuaciones diferenciales parciales no lineales utilizando un método numérico sencillo (de diferencias finitas) implementado en una herramienta computacional versátil y de fácil utilización por usuarios no iniciados. Esto permite que la explicación del uso de la herramienta no se lleve más tiempo que el planteamiento del fenómeno físico que se estudia.

Como ejemplo de aplicación, se resolvió el sistema de ecuaciones del modelo macroscópico de tráfico vehicular de Greenberg, el cual se deduce de las ecuaciones de NavierStokes y continuidad que representan el movimiento de un fluido. En este caso se supone el flujo como compresible [23].

Para tener datos reales y usarlos como datos de entrada del modelo, se realizó un estudio de tráfico vehicular en una vialidad de la Ciudad de México. Se midió la densidad vehicular, el flujo vehicular y la velocidad. Se tomó una sección de una avenida importante (en años pasados aún era un área sin urbanizar) de alrededor de $150 \mathrm{~m}$ de largo y se observó por aproximadamente $3 \mathrm{hrs}$. Las variables calculadas con los datos tomados en campo tuvieron un error de medición máximo de $5.3 \%$, el cual es aceptable para los propósitos de este trabajo. Posteriormente, se realizó un análisis de datos siguiendo el procedimiento de Greenberg [16], con el propósito de calcular el parámetro $c$ que se usó como dato de entrada en el modelo numérico.

Se utiliza un método numérico para resolver el sistema completo (bajo el supuesto de que la densidad es una función de la velocidad), tanto en el caso con la ecuación de continuidad usual, como con la ecuación de continuidad modificada. Las condiciones de frontera son las variables medidas en el lugar de estudio y la derivada normal igualada a cero.

Al evaluar directamente el modelo de tráfico original de Greenberg, resultó que la densidad se mantenía constante en tiempo y espacio, y los valores de velocidad no representan, en general, cantidades reales. En el mismo ejercicio se propuso que esto se debe a que en ese segmento de vialidad no se cumple el principio de conservación de la masa, por lo tanto la ecuación de continuidad no es homogénea. Al calcular el término no homogéneo a partir de los datos medidos y usarlo como dato de entrada en la simulación, los resultados mejoraron al observar que los valores para la densidad y la velocidad fueron más congruentes con la realidad, pero las velocidades negativas seguían existiendo. 
Asimismo, se realizaron simulaciones modificando el modelo y considerando el término de viscosidad. Dado que el modelo se construyó a partir de las ecuaciones de NavierStokes para un flujo compresible, la viscosidad utilizada representa a la viscosidad dinámica, pues en este caso la viscosidad cinemática se modificará conforme varíe la densidad $k(x, t)$. Esta es la razón por la cual si la viscosidad aumenta, la velocidad también es más grande.

El modelo del tráfico se puede complicar tanto como se quiera y la metodología puede ser la misma. El proponer este tipo de ejercicios, que involucran la realización de experimentos sencillos el contraste con simulaciones numéricas enriquece la enseñanza y motiva al alumno a conocer herramientas computacionales, mismas que los alumnos de física y matemáticas piensan que no necesitan.

Por otro lado, aprender el uso de lenguajes de programación de alto nivel, y de libre distribución como $\mathrm{C} / \mathrm{C}++$, Fortran, Pascal, entre otros, por parte de los alumnos, requiere tiempo que muy difícilmente se tiene en un semestre académico. Los planes de estudio de las licenciaturas aquí contempladas generalmente no incluyen un curso de programación y las asignaturas concernientes a computación y métodos numéricos tiene una carga de contenidos que deben cumplirse, por lo cual los estudiantes pueden emplear cualquier herramienta a condición que la conozcan lo suficiente para realizar las actividades. Esto representa un problema en carreras científicas que no se especializan en computación, pues los planes de estudio consideran muy pocas materias en donde les enseñen a los alumnos lenguajes de programación.

Existen programas comerciales como Matlab ${ }^{\mathrm{TM}}$ [36] que pueden ayudar a solventar esta deficiencia, los cuales son más amigables para el aprendizaje, pues tienen una gran variedad de bibliotecas, subrutinas, procedimientos y funciones intrínsecas, además de que la ayuda integrada posee un gran número de ejemplos. Sin embargo, las licencias de dichos programas comerciales son muy caras y aunque ya se empieza a promover la compra de licencias estudiantiles por parte de las instituciones educativas de nivel superior, no todas destinan presupuesto para ese rubro. Es por eso que se propone utilizar software libre.

Scilab es un programa de libre distribución, muy parecido a Matlab pero mucho más ligero. Es más amigable para la mayoría de los estudiantes que lenguajes como Fortran y $\mathrm{C} / \mathrm{C}++$. La sintaxis es parecida a la de Matlab y los datos generados se pueden graficar directamente, sin necesidad de guardarlos en archivos de texto para después leerlos con un graficador. Ciertamente, también existe una instrucción para escribir los datos en archivos de texto, en caso de que se requiera.

Por todo lo anterior, en las clases se empezó a promocionar este lenguaje de programación a los alumnos. Algunos estudiantes prefirieron quedarse con los programas que dominaban (más o menos un $10 \%$ del grupo), entre ellos Julia, Python y Java. Otros alumnos que no sabían programar, se quedaron con Excel y lápiz y papel, aunque eran una minoría (aproximadamente un $5 \%$ ). Pero la mayoría probó Scilab (incluyendo algunos que dominaban otros lenguajes de programación de alto nivel) lo eligieron para sus tareas escolares en las asignaturas concernientes a computación y métodos numéricos.

Generalmente, las clases de métodos numéricos, computación, física computacional, etc. son sesiones de tres horas, lo que permite explicar el planteamiento del problema, el desarrollo del algoritmo y la implementación (escritura del código numérico y depuración de errores). Con los programas como Fortran y $\mathrm{C} / \mathrm{C}++$, se necesitaban de 3 a 5 sesiones para explicar el uso de esos lenguajes de programación, la implementación del código numérico, depuración de errores y graficación de los datos obtenidos. Con Scilab se requieren de 2 a 3 sesiones, y se obtiene en general un mejor desempeño por parte de los alumnos.

Desde luego, se les advierte a los alumnos sobre la eficacia y eficiencia de dichos lenguajes de programación. Por ejemplo, los estudiantes de séptimo semestre realizaron un ejercicio sobre la obtención del perfil parabólico de un flujo estacionario en un canal haciendo una prueba de tiempo de ejecución, donde se comparó Scilab con Fortran. Para integrar la ecuación (usando métodos espectrales de Chebyshev), en igualdad de condiciones, Scilab tardó $12 \mathrm{~s}$, mientras que Fortran se llevó solo $2.5 \mathrm{~s}$. Algo similar ocurre al comparar un programa ejecutado en Matlab y $\mathrm{C}$, a pesar de que Matlab está escrito en C. No obstante, para docencia y ejercicios en clase, el hecho que la ejecución de un programa tarde de $20 \mathrm{~s}$ a 2 min es aceptable.

En este ejercicio numérico del tráfico vehicular en Scilab, el programa tarda menos de dos minutos en ejecutarse (corriendo en Linux en una computadora portátil estándar, de 2 núcleos y memoria RAM de $8 \mathrm{~Gb}$ ), utilizando una tolerancia de $1 \times 10^{-10}$, empleada en investigación científica. Los alumnos pueden usar tolerancias más grandes. La experiencia docente indica que entre $1 \times 10^{-4}$ y $1 \times 10^{-6}$ (se usa menos tiempo de cálculo que con tolerancias de $10^{-10}$ ) es suficiente para lograr el objetivo docente: entender el problema físico y el funcionamiento de la herramienta computacional. El número de Courant indicó la convergencia numérica del método. Cabe mencionar que todas las gráficas presentadas en este trabajo (salvo la Fig. 4) son las que produce el programa Scilab.

Existen alumnos más avanzados en programación que otros, así que ellos pueden elegir el lenguaje de programación que más les guste para la implementación. Existen otros menos aventajados, que por el tiempo o porque simplemente no resulta de su interés, no aprenden a programar y prefieren utilizar las hojas de cálculo. Scilab puede ayudar al relativamente rápido aprendizaje de la programación estructurada para la pronta implementación de los códigos numéricos vistos en clases. 


\section{Agradecimientos}

A los revisores anónimos, cuyos comentarios y sugerencias mejoraron notablemente el artículo. E. J. López-Sánchez agradece al Programa de Becas Posdoctorales DGAPAUNAM 2018-2020. Los autores agradecen al SNI.
1. Facultad de Ciencias, UNAM, Plan de Estudios de la Licenciatura en Física. http://www. fciencias.unam.mx/ licenciatura/mapa/106/1081

2. Facultad de Ciencias, UNAM, Plan de Estudios de la Licenciatura en Física Biomédica. http://www. fciencias. unam.mx/licenciatura/mapa/134/2016

3. Facultad de Ingeniería, UNAM. Plan de Estudios, Ingeniería Civil. http://www.fciencias.unam.mx/ licenciatura/mapa/106/1081

4. Universidad Autónoma Metropolitana, Unidad Iztapalapa, Plan de Estudios de la Licenciatura en Física. https://www.uam.mx/licenciaturas/pdfs/ 26_7b_Licenciatura_en_Fisica_IZT.pdf

5. Escuela Superior de Física y Matemáticas, IPN. Plan de Estudios de la Licenciatura en Física y Matemáticas. https:// www.esfm.ipn.mx/unidades-aprendizaje.html

6. Universidad Autónoma Metropolitana, Unidad Azcapotzalco. Plan de Estudios de la Licenciatura en Ingeniería Física. http://www.uam.mx/licenciaturas/pdfs/ 4_15_Lic_en_Ingenieria_Fisica_AZC.pdf

7. Centro Universitario de Ciencias Exactas e Ingeniería, U. de G. Plan de Estudios de la Licenciatura en Física. http://www.cucei.udg.mx/carreras/fisica/ sites/default/files/dictamenfis2012.pdf

8. Universidad de Sonora, Plan de Estudios de la Licenciatura en Física. http://licfis.fisica.uson.mx/ licplan.html

9. Instituto de Estudios Superiores de Monterrey, Plan de Estudios de la Licenciatura en Ingeniería Física Industrial https://admision.itesm.mx/es/ ingenieria-cienciasaplicadas/ifi

10. S. I. Grossman S. y J. J. Flores G. Álgebra lineal Mc Graw Hill $7 \mathrm{ma}$ ed. México D. F. (2012).

11. B. D. Greenshields, A study of highway capacity. Proceedings Highway Research Record. 14 (1935) 448-477

12. F. van Wageningen-Kessels, $H$. van Lint $K$. Vuik $y$ S. Hoogendoorn, Genealogy of traffic flow models. $E U$ RO J. Transp Logist 4 (2015) 445-473 DOI:10.1007/ s13676-014-0045-5

13. C. F. Daganzo, M. J. Cassidy, R. L. Bestini, Possible explanations of phase transitions in highway traffic. Transportation Resarch Part A. 33 (1999) 365-379. https : //doi.org/10. 1016/S0965-8564(98)00034-2

14. R. M. Velasco and W. Marquez, Navier-Stokes-like equations for traffic flow. Phys. Rev. E. 72 (2005) 046102. DOI: 10. 1103/PhysRevE.72.046102

15. ESI-Group. SCILAB 6.0.1 (2018) http://www.scilab. org
16. H. Greenberg, An Analysis of Traffic Flow. Operations Research. 7 (1959) 79-85. https://doi.org/10.1287/ opre.7.1.79

17. O. A. Rosas-Jaimes, L. A. Quezada-Téllez and G. FernándezAnaya, Stability analysis in Greenberg's traffic model. Inter. $J$. Mod. Phys. C. 28 (2017) 1750130. https : / / doi.org/10. $1142 / \mathrm{S} 0129183117501303$

18. R. Haberman, Mathematical Models Mechanical Vibrations, Population Dynamics, and Traffic Flow. An Introduction to Applied Mathematics SIAM, Philadelphia, (USA 1998).

19. N. H. Asmar, Partial differential equations with Fourier series and boundary value problems. Pearson, (Prentice Hall. USA 2005).

20. H. J. Weber and G. B. Arfken, Essential Mathematical Methods for Physicists. Academic Press, USA (2003).

21. M. L. Boas. Mathematical methods in the physical sciences. (2nd Ed. John Wiley \& Sons. USA 1983).

22. J. Delgado, P. Saavedra y R.M. Velasco. Tráfico Vehicular. Publicaciones de CBI UAM-Iztapalapa, México (2012).

23. E. Guyon, J-P. Hulin, L. Petit and C. D. Mitescu. Physical Hydrodynamics. Oxford University Press. N. Y. USA (2001).

24. F. M. White. Mecánica de Fluidos. Mc Graw Hill. Madrid, España (2004).

25. E. Gómez-Hernández, Desarrollo de un Modelo de Simulación Vehicular para la Mejora en la Sincronización de Semáforos. Tesis Doctoral. INAOE, Puebla, México (2009).

26. Google Earth. Google Earth Pro. https://www.google. com/intl/es/earth/download/gep/agree.html

27. B. Oda-Noda. Introducción al Análisis Gráfico de Datos Experimentales. Las prensas de Ciencias. Facultad de Ciencias, UNAM. México (2005)

28. F. Kessels, Traffic Flow Modelling. Introduction to Traffic Flow Theory Through a Genealogy of Models. Springer. Cham, Switzerland (2019).

29. B. S. Kerner and P. Konhäuser, Structure and parameters of clusters in traffic flow. Phys. Rev. E 50 (1994) 54-83. https : //doi.org/10.1103/PhysRevE.5054

30. R. L. Burden and J. D. Faires, Análisis numérico. Thomson Learning. México (2002). 
31. J. H. Matthews and K. D. Fink. Numerical Methods: Using Matlab. Prentice Hall 3rd edition. USA (1999).

32. S. C. Chapra y R. P. Canale. Métodos numéricos para ingenieros. 6a. edición McGraw-Hill México.

33. W. Cheney y D. Kincaid. Métodos numéricos y computación. 6a. edición, Cengage Learning. México, (2011)

34. M. Treiber and A. Kesting, A. Traffic Flow Dynamics: Data,
Models and Simulation. Springer-Verlag. Berlin-Heildelgerg (2013).

35. ESI-Open CFD OpenFOAM, The Open Source CFD Toolbox. Programmer's Guide. Version 2.4. (2015) https:// openfoam.org.

36. MathWorks ${ }^{\circledR}$. Matlab. https://www. mathworks.com/ products/matlab.html 